\title{
Patient Experience Connecting Mobile-Based Self-Monitoring of Diet and Physical Activity to Diabetes Educators through a Connected Interface in an Electronic System for Diabetes Education
}

\author{
Jing Wang ${ }^{1}$, PhD, MPH, RN, FAAN; Brittney Lewis ${ }^{1}$, MS, NCC; Linda Siminerio ${ }^{2}$, PhD, CDE, RN, FAAN
}

${ }^{1}$ School of Nursing, University of Texas Health Science Center at San Antonio, San Antonio, TX, United States

${ }^{2}$ School of Medicine, University of Pittsburgh, Pittsburgh, PA, United States

Corresponding Author:

Jing Wang, PhD, MPH, RN, FAAN

School of Nursing

University of Texas Health Science Center at San Antonio

7703 Floyd Curl Drive

San Antonio, TX, 78229-3900

United States

Phone: 12104508561

Email: wangj1@uthscsa.edu

\section{Abstract}

Background: Smartphone applications and wearable activity trackers have become popular tools in recent years in managing chronic diseases such as diabetes. More recently, studies have focused on connecting patient-generated health data from mobile devices directly to health care providers and educators. However, not much is known regarding the patient experience in using these mobile devices for diabetes management, particularly the implications of allowing educators direct access to patients' diet and exercise data.

Objective: The objective of this study was to identify patients' perceived benefits and concerns about using a smartphone application and wristband activity tracker to monitor diet and physical activity, as well as the perceived benefits and concerns of allowing educators access to such data.

Methods: We conducted a qualitative, descriptive study as an axillary study to a clinical trial testing a connected interface to link patient self-monitoring diet and physical activity to a nationally used electronic diabetes education system. Our axillary study examined 13 type 2 diabetes patients' views on perceived benefits and concerns about using a smartphone application and wristband activity tracker to monitor diet and physical activity for three months. A focus group interview was administered to obtain general and specific understanding of the use of smartphone applications and activity trackers during the study period. The central interview questions guiding the discussion included "What did you think about the UP24 wristband and app?", "What are your thoughts about the connection of UP24 data with Chronicle, the Web-based diabetes education system, so that your diabetes educators can see your behavior?", and "Has knowing that someone else has access to your diet and exercise data affected your behavior and self-monitoring?" The interviewer also asked specific questions to gain deeper understanding of the following topics: (1) the app and wristband features used to record and monitor diet and physical activity, (2) materials used for intervention orientation, (3) additional data (eg, weight and blood glucose) that participants would like to share with educators, and (4) suggestions for improvement in diabetes self-management and communication with educators and physicians. The focus group sessions were audio-recorded and transcribed. Transcribed data were analyzed to identify key themes based on interpretive coding procedures.

Results: We identified 11 key themes under three major categories and described these themes with illustrative quotations. The three major categories of themes covered (1) self-monitoring themes: varied experience and self-monitoring patterns and adherence exist among patients using the wearable tracker and its companion smartphone application; (2) themes related to sharing self-monitoring of diet and physical activity data with diabetes educators: sharing self-monitoring diet influences patient self-monitoring adherence and dietary and activity changes, and communication with educators; and (3) research study-related themes: technical barriers, utilization of manuals and tutorial videos in beginning use of the connected health tools, and desired features on combining lifestyle data with glucose data and caregiver access. 
Conclusions: Connected technology aiming to incorporate patient-generated health lifestyle data into clinical workflow should consider patient perspectives in terms of their experience and motivation for generating and sharing such data and technical barriers in using such tools.

(iproc 2018;4(2):e11905) doi: $10.2196 / 11905$

\section{KEYWORDS}

activity monitoring; chronic disease; chronic disease management; dietary assessment; mobile health; mobile technology; diabetes

Edited by T Hale; this is a non-peer-reviewed article. Submitted 09.08.18; accepted 29.08.18; published 17.09.18.

Please cite as:

Wang J, Lewis B, Siminerio L

Patient Experience Connecting Mobile-Based Self-Monitoring of Diet and Physical Activity to Diabetes Educators through a Connected Interface in an Electronic System for Diabetes Education

iproc 2018;4(2):e11905

URL: http://www.iproc.org/2018/2/e11905/

doi: $10.2196 / 11905$

PMID:

(CJing Wang, Brittney Lewis, Linda Siminerio. Originally published in Iproceedings (http://www.iproc.org), 17.09.2018. This is an open-access article distributed under the terms of the Creative Commons Attribution License (https://creativecommons.org/licenses/by/4.0/), which permits unrestricted use, distribution, and reproduction in any medium, provided the original work, first published in Iproceedings, is properly cited. The complete bibliographic information, a link to the original publication on http://www.iproc.org/, as well as this copyright and license information must be included. 